\title{
Erratum: A complete survey of texture zeros in the lepton mass matrices
}

\author{
P.O. Ludl and W. Grimus \\ University of Vienna, Faculty of Physics \\ Boltzmanngasse 5, A-1090 Vienna, Austria \\ E-mail: patrick.ludl@univie.ac.at, walter.grimus@univie.ac.at
}

ERRATUM TO: JHEP07(2014)090

ARXIV EPRINT: 1406.3546

Corrections in the numerical analysis: there was an error in the algorithm we had used to remove phases in the mass matrices by means of weak-basis transformations. As a result, in some cases too many phases had been removed. Furthermore, there was an error in the numerical computation of the mixing matrix, which affected the analysis of some of the models. We have corrected both errors in our programs and have repeated all numerical computations. The new results can be found in tables 9 to 13 which replace the corresponding tables in the paper.

Consequences of the new analysis for the conclusions: though the set of textures compatible with experiment and the set of maximally restrictive textures have changed, the effect on the conclusions is small — see tables 9 to 13.

In the case of Dirac neutrinos, there is only one relevant change, namely in the predictive power of the maximally restrictive textures with respect to the Dirac phase $\delta$. Originally, since in general too many phases had been removed from the mass matrices, $\delta$ was forced to be exactly 0 or $\pi$. The correct rephasing leads to $\delta^{\text {min }} \approx 0$ and $\delta^{\text {max }} \approx 2 \pi$ for all but one of the textures - see the captions of tables 10 and 11 .

In the case of Majorana neutrinos, there is one notable change, namely in the predictivity of the texture $\left(M_{\ell}, M_{L}\right) \sim\left(6_{1}^{(\ell)}, 2_{1}^{\left(\nu_{L}\right)}\right)$ with an inverted neutrino mass spectrum. The corrected analysis gives $\Delta\left(\sin ^{2} \theta_{23}\right)=53.8<100$ and this case is thus predictive with respect to $\sin ^{2} \theta_{23}$ according to the criterion of equation (5.6) in the paper. However, tightening this criterion to $\Delta\left(\mathcal{O}_{i}\right) \leq 25$, i.e. from $10 \sigma$ to $5 \sigma$, there is still no texture which can predict any of the charged-lepton masses or the neutrino oscillation parameters.

Open Access. This article is distributed under the terms of the Creative Commons Attribution License (CC-BY 4.0), which permits any use, distribution and reproduction in any medium, provided the original author(s) and source are credited. 


\begin{tabular}{|l|c|c|c|c|}
\hline Neutrino nature & \multicolumn{2}{|c|}{ Dirac } & \multicolumn{2}{c|}{ Majorana } \\
\hline Neutrino mass spectrum & normal & inverted & normal & inverted \\
\hline Number of textures & 570 & 570 & 298 & 298 \\
\hline Compatible with experiment & 430 & 429 & 215 & 229 \\
\hline Compatible and maximally restrictive & 29 & 28 & 27 & 25 \\
\hline
\end{tabular}

Table 9. Results of the $\chi^{2}$-analysis.

\begin{tabular}{|c|c|c|c|c|}
\hline$\left(M_{\ell}, M_{D}\right)$ & $n$ & $m_{0}^{\min }[\mathrm{eV}]$ & $m_{0}^{\max }[\mathrm{eV}]$ & texture predicts \\
\hline $3_{2}-7_{1}$ & 9 & $0.00 \times 10^{0}$ & $0.00 \times 10^{0}$ & $m_{0}$ \\
$3_{2}-7_{3}$ & 9 & $0.00 \times 10^{0}$ & $0.00 \times 10^{0}$ & $m_{0}$ \\
$4_{1}-6_{1}$ & 9 & $0.00 \times 10^{0}$ & $0.00 \times 10^{0}$ & $m_{0}$ \\
$4_{1}-6_{3}$ & 9 & $0.00 \times 10^{0}$ & $0.00 \times 10^{0}$ & $m_{0}$ \\
$4_{1}-6_{4}$ & 9 & $0.00 \times 10^{0}$ & $0.00 \times 10^{0}$ & $m_{0}$ \\
$4_{1}-6_{5}$ & 9 & $0.00 \times 10^{0}$ & $0.00 \times 10^{0}$ & $m_{0}$ \\
$4_{1}-6_{6}$ & 9 & $0.00 \times 10^{0}$ & $0.00 \times 10^{0}$ & $m_{0}$ \\
$4_{2}-6_{1}$ & 9 & $0.00 \times 10^{0}$ & $0.00 \times 10^{0}$ & $m_{0}$ \\
$4_{2}-6_{2}$ & 9 & $0.00 \times 10^{0}$ & $0.00 \times 10^{0}$ & $m_{0}$ \\
$4_{2}-6_{3}$ & 9 & $0.00 \times 10^{0}$ & $0.00 \times 10^{0}$ & $m_{0}$ \\
$4_{2}-6_{7}$ & 9 & $0.00 \times 10^{0}$ & $0.00 \times 10^{0}$ & $m_{0}$ \\
$4_{2}-6_{8}$ & 9 & $0.00 \times 10^{0}$ & $0.00 \times 10^{0}$ & $m_{0}$ \\
$4_{3}-6_{1}$ & 9 & $0.00 \times 10^{0}$ & $0.00 \times 10^{0}$ & $m_{0}$ \\
$4_{3}-6_{2}$ & 9 & $0.00 \times 10^{0}$ & $0.00 \times 10^{0}$ & $m_{0}$ \\
$4_{3}-6_{3}$ & 9 & $0.00 \times 10^{0}$ & $0.00 \times 10^{0}$ & $m_{0}$ \\
$4_{3}-6_{4}$ & 9 & $0.00 \times 10^{0}$ & $0.00 \times 10^{0}$ & $m_{0}$ \\
$4_{3}-6_{5}$ & 9 & $0.00 \times 10^{0}$ & $0.00 \times 10^{0}$ & $m_{0}$ \\
$4_{3}-6_{6}$ & 9 & $0.00 \times 10^{0}$ & $0.00 \times 10^{0}$ & $m_{0}$ \\
$4_{3}-6_{7}$ & 9 & $0.00 \times 10^{0}$ & $0.00 \times 10^{0}$ & $m_{0}$ \\
$4_{3}-6_{8}$ & 9 & $0.00 \times 10^{0}$ & $0.00 \times 10^{0}$ & $m_{0}$ \\
$4_{3}-6_{9}$ & 9 & $0.00 \times 10^{0}$ & $0.00 \times 10^{0}$ & $m_{0}$ \\
$5_{1}-5_{1}$ & 9 & $0.00 \times 10^{0}$ & $0.00 \times 10^{0}$ & $m_{0}$ \\
$5_{1}-5_{4}$ & 9 & $0.00 \times 10^{0}$ & $0.00 \times 10^{0}$ & $m_{0}$ \\
$5_{1}-5_{5}$ & 9 & $0.00 \times 10^{0}$ & $0.00 \times 10^{0}$ & $m_{0}$ \\
$5_{1}-5_{6}$ & 9 & $0.00 \times 10^{0}$ & $0.00 \times 10^{0}$ & $m_{0}$ \\
$5_{1}-5_{8}$ & 9 & $0.00 \times 10^{0}$ & $0.00 \times 10^{0}$ & $m_{0}$ \\
$6_{1}-3_{12}$ & 10 & $1.69 \times 10^{-2}$ & $3.32 \times 10^{-1}$ & - \\
$6_{1}-4_{1}$ & 9 & $0.00 \times 10^{0}$ & $0.00 \times 10^{0}$ & $m_{0}$ \\
$6_{1}-4_{5}$ & 8 & $1.15 \times 10^{-2}$ & $1.65 \times 10^{-2}$ & $m_{0}$ \\
\hline & & & & \\
\hline
\end{tabular}

Table 10. The maximally restrictive and compatible classes of texture zeros in $\left(M_{\ell}, M_{D}\right)$. The number of physical parameters of the texture is denoted by $n$. For all textures in this table $\chi_{\min }^{2}<$ $10^{-4}$. For the Dirac phase $\delta$ one finds $\delta^{\min } \approx 0$ and $\delta^{\max } \approx 2 \pi$ for all textures except $6_{1}-4_{5}$, where $\delta=0$ or $\pi$. Part 1: normal neutrino mass spectrum. 


\begin{tabular}{|c|c|c|c|c|}
\hline$\left(M_{\ell}, M_{D}\right)$ & $n$ & $m_{0}^{\min }[\mathrm{eV}]$ & $m_{0}^{\max }[\mathrm{eV}]$ & texture predicts \\
\hline $3_{2}-7_{1}$ & 9 & $0.00 \times 10^{0}$ & $0.00 \times 10^{0}$ & $m_{0}$ \\
$3_{2}-7_{3}$ & 9 & $0.00 \times 10^{0}$ & $0.00 \times 10^{0}$ & $m_{0}$ \\
$4_{1}-6_{1}$ & 9 & $0.00 \times 10^{0}$ & $0.00 \times 10^{0}$ & $m_{0}$ \\
$4_{1}-6_{3}$ & 9 & $0.00 \times 10^{0}$ & $0.00 \times 10^{0}$ & $m_{0}$ \\
$4_{1}-6_{4}$ & 9 & $0.00 \times 10^{0}$ & $0.00 \times 10^{0}$ & $m_{0}$ \\
$4_{1}-6_{5}$ & 9 & $0.00 \times 10^{0}$ & $0.00 \times 10^{0}$ & $m_{0}$ \\
$4_{1}-6_{6}$ & 9 & $0.00 \times 10^{0}$ & $0.00 \times 10^{0}$ & $m_{0}$ \\
$4_{2}-6_{1}$ & 9 & $0.00 \times 10^{0}$ & $0.00 \times 10^{0}$ & $m_{0}$ \\
$4_{2}-6_{2}$ & 9 & $0.00 \times 10^{0}$ & $0.00 \times 10^{0}$ & $m_{0}$ \\
$4_{2}-6_{3}$ & 9 & $0.00 \times 10^{0}$ & $0.00 \times 10^{0}$ & $m_{0}$ \\
$4_{2}-6_{7}$ & 9 & $0.00 \times 10^{0}$ & $0.00 \times 10^{0}$ & $m_{0}$ \\
$4_{2}-6_{8}$ & 9 & $0.00 \times 10^{0}$ & $0.00 \times 10^{0}$ & $m_{0}$ \\
$4_{3}-6_{1}$ & 9 & $0.00 \times 10^{0}$ & $0.00 \times 10^{0}$ & $m_{0}$ \\
$4_{3}-6_{2}$ & 9 & $0.00 \times 10^{0}$ & $0.00 \times 10^{0}$ & $m_{0}$ \\
$4_{3}-6_{3}$ & 9 & $0.00 \times 10^{0}$ & $0.00 \times 10^{0}$ & $m_{0}$ \\
$4_{3}-6_{4}$ & 9 & $0.00 \times 10^{0}$ & $0.00 \times 10^{0}$ & $m_{0}$ \\
$4_{3}-6_{5}$ & 9 & $0.00 \times 10^{0}$ & $0.00 \times 10^{0}$ & $m_{0}$ \\
$4_{3}-6_{6}$ & 9 & $0.00 \times 10^{0}$ & $0.00 \times 10^{0}$ & $m_{0}$ \\
$4_{3}-6_{7}$ & 9 & $0.00 \times 10^{0}$ & $0.00 \times 10^{0}$ & $m_{0}$ \\
$4_{3}-6_{8}$ & 9 & $0.00 \times 10^{0}$ & $0.00 \times 10^{0}$ & $m_{0}$ \\
$4_{3}-6_{9}$ & 9 & $0.00 \times 10^{0}$ & $0.00 \times 10^{0}$ & $m_{0}$ \\
$5_{1}-5_{1}$ & 9 & $0.00 \times 10^{0}$ & $0.00 \times 10^{0}$ & $m_{0}$ \\
$5_{1}-5_{4}$ & 9 & $0.00 \times 10^{0}$ & $0.00 \times 10^{0}$ & $m_{0}$ \\
$5_{1}-5_{5}$ & 9 & $0.00 \times 10^{0}$ & $0.00 \times 10^{0}$ & $m_{0}$ \\
$5_{1}-5_{6}$ & 9 & $0.00 \times 10^{0}$ & $0.00 \times 10^{0}$ & $m_{0}$ \\
$5_{1}-5_{8}$ & 9 & $0.00 \times 10^{0}$ & $0.00 \times 10^{0}$ & $m_{0}$ \\
$6_{1}-3_{12}$ & 10 & $<10^{-3}$ & $3.31 \times 10^{-1}$ & - \\
$6_{1}-4_{1}$ & 9 & $0.00 \times 10^{0}$ & $0.00 \times 10^{0}$ & $m_{0}$ \\
\hline & & & & \\
\hline
\end{tabular}

Table 11. The maximally restrictive and compatible classes of texture zeros in $\left(M_{\ell}, M_{D}\right)$. The number of physical parameters of the texture is denoted by $n$. For all textures in this table $\chi_{\min }^{2}<$ $10^{-4}, \delta^{\min } \approx 0$ and $\delta^{\max } \approx 2 \pi$. Part 2 : inverted neutrino mass spectrum. 


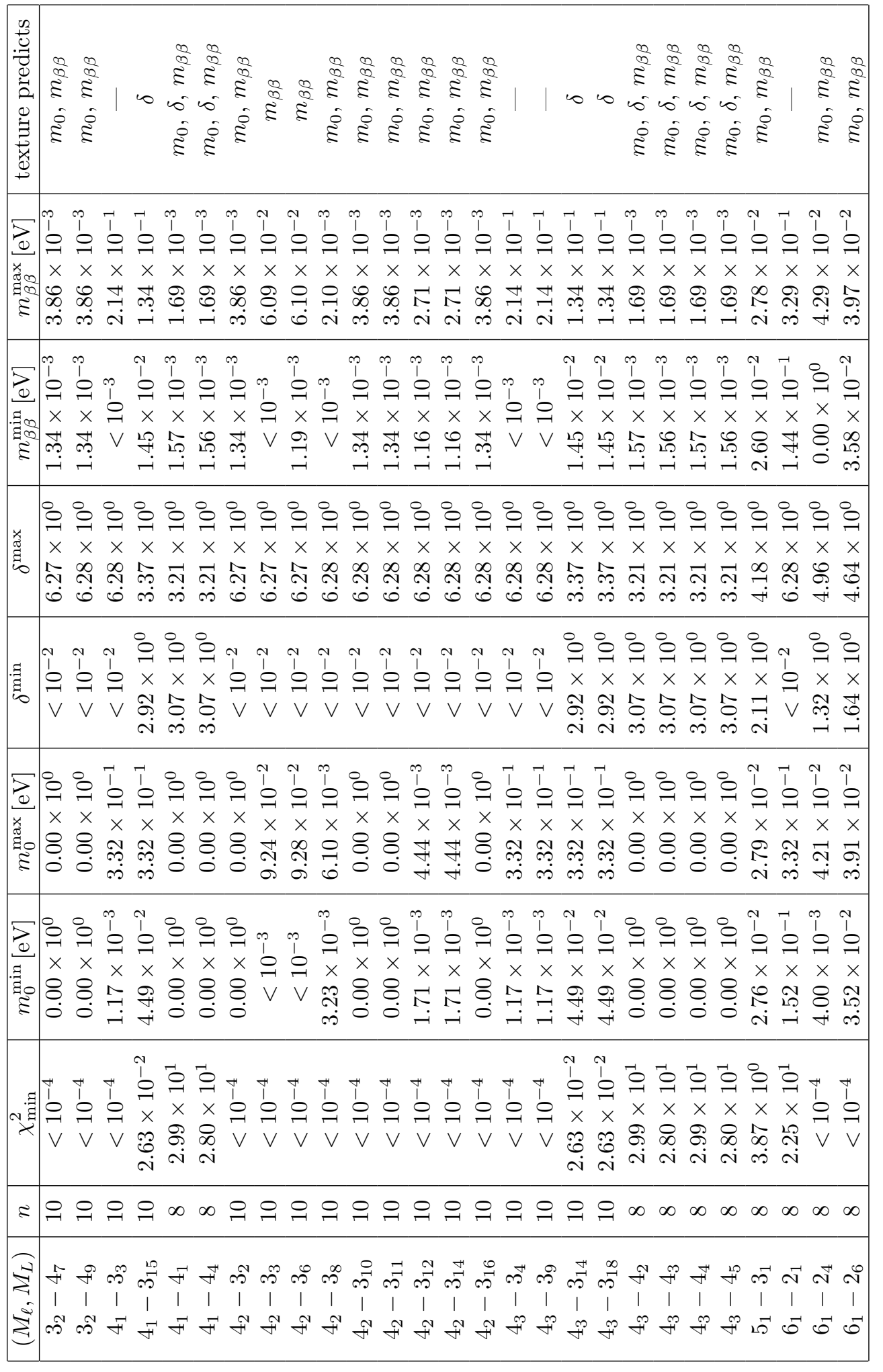




\begin{tabular}{|c|c|}
\hline & 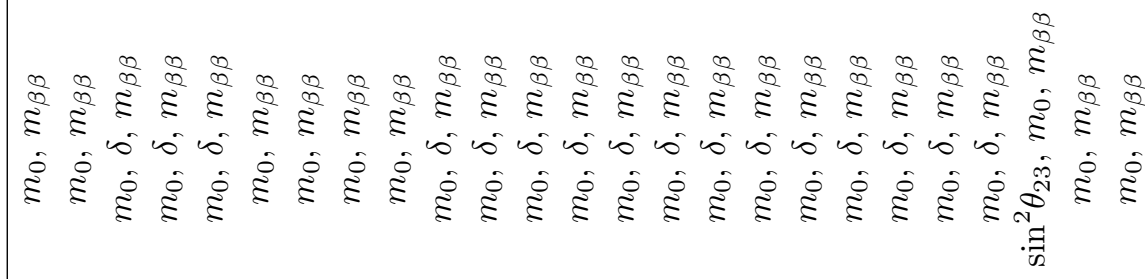 \\
\hline & 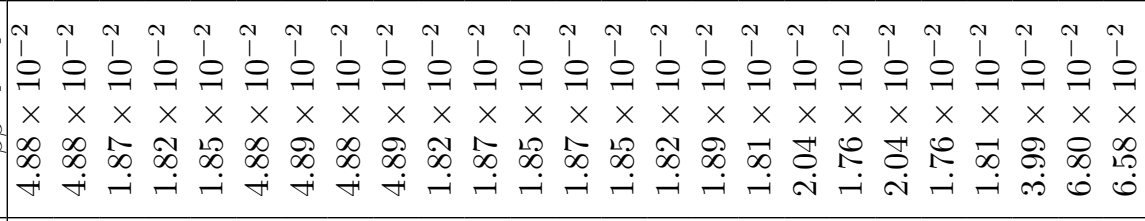 \\
\hline & 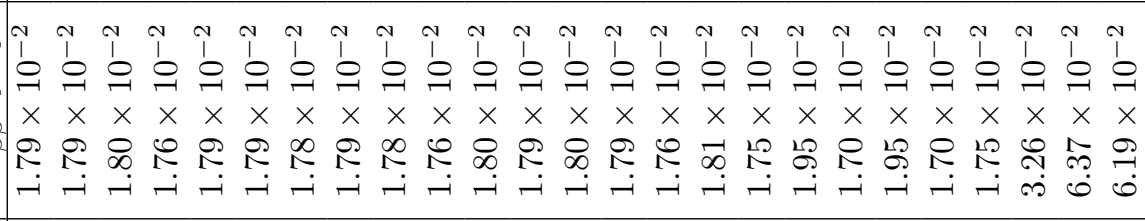 \\
\hline & 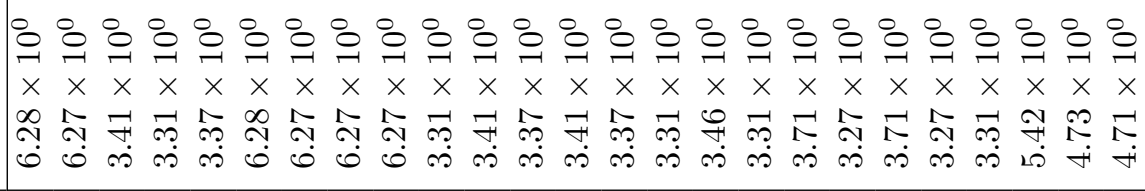 \\
\hline & 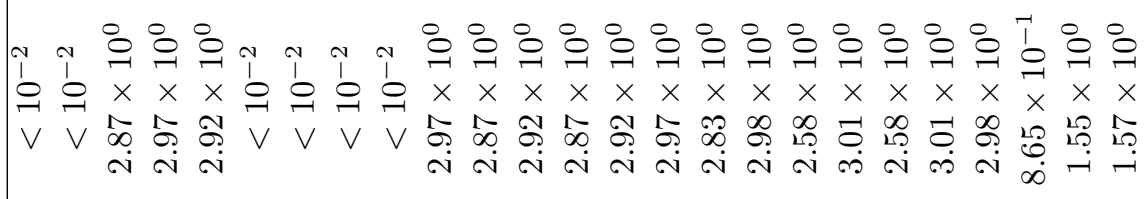 \\
\hline & 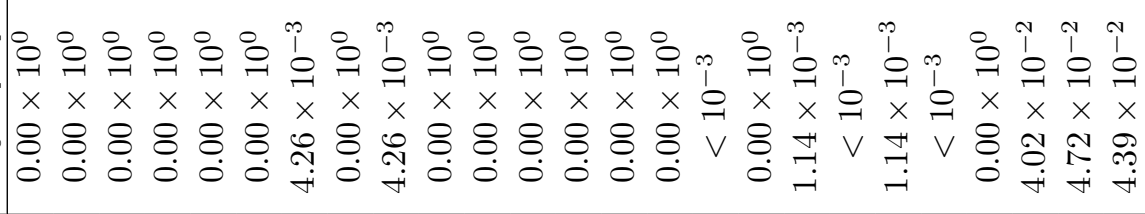 \\
\hline & 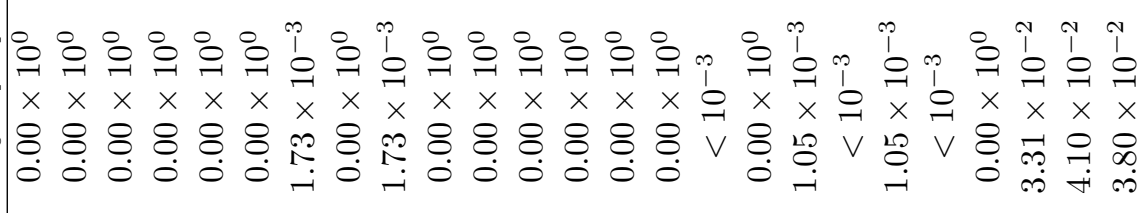 \\
\hline & 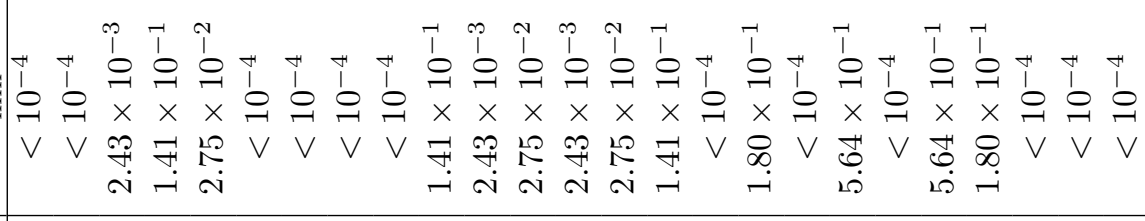 \\
\hline 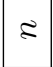 & 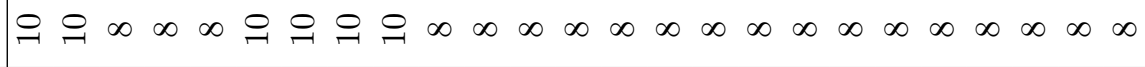 \\
\hline & 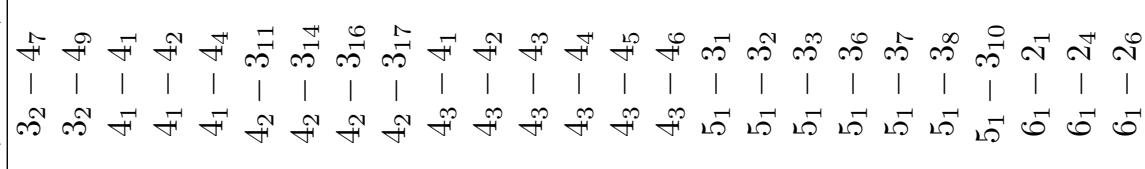 \\
\hline
\end{tabular}

